\section{Experiments and volunteers}

SIR - I should like to respond to your News item "Nerve gas volunteers demand follow-up study" (Nature 367, 303; 1994).

The role of the Chemical and Biological Defence Establishment (CBDE) at Porton Down is to ensure that the UK Armed Forces have effective protective measures against the threat that chemical or biological weapons may be used against them. In order to carry out this work, it is necessary to use Service volunteers to assess the ability of Service personnel to function with new equipment and procedures; to develop medical countermeasures to protect Service personnel; and to evaluate the effects of very low and medically safe concentrations of chemical warfare agents on the ability of unprotected personnel to operate normally.

No studies involving volunteers are carried out unless there is a clear military need and a detailed protocol has been reviewed and approved by an independent ethics committee in accordance with the guidelines laid down by the Royal College of Physicians.

The procedure is that, on arrival at CBDE, Service volunteers are given a thorough medical examination to ensure that they are fit for the proposed study and are not suffering from any condition that might prejudice its results. The nature of the study is explained to them and they are told that they may withdraw without explanation at any stage and without detriment, penalty or pressure. The explanation about the study is given by those conducting it and by a military officer in a lay statement, in words that the volunteers will understand. They are advised how they may receive compensation in the unlikely event of their suffering any personal injury as a consequence of their having been volunteers at CBDE.

At the end of the study, the volunteers are given a second medical examination and the fact that they have participated at CBDE in a volunteer study is recorded on their medical records.

The practice has recently been instituted in which the lay statement read to all volunteers by a military officer states that "It is CBDE policy to call back some volunteer subjects for re-testing from time to time to ensure that techniques used give consistent and reproducible results and that no changes in the way we have applied the tests have occurred with time." From time to time, Service volunteers have been recalled so that checks on their medical health can be made. There is no particular frequency or pattern to such recalls. In addition, some volunteers return voluntarily to CBDE to take part in subsequent unrelated studies. The recall of any volunteers to CBDE is noted on their medical records.
When people leave the Services, their medical records are retained by the Ministry of Defence, but they are made available on request to the individual former serviceman or woman's doctor should the doctor wish to have access to them.

It is not in the national interest to publish details of volunteer studies as this information could be of significant benefit to a potential aggressor as he would be aware of the current state of our work to develop improved protective measures. However, during the past 30 years, the only nerve agent to which volunteers have been exposed at very low and medically safe concentrations was sarin, GB. Over the past 30 years, there has been no evidence that the health of Service volunteers has deteriorated as a result of their participation as volunteers at CBDE Porton Down. There are, therefore, no plans to recall volunteers other than those referred to above.

\section{Graham S. Pearson}

(Director General)

Ministry of Defence,

Chemical \& Biological

Defence Establishment,

Porton Down, Salisbury, Wilts SP4 OJQ, UK

\section{Cyclotron future}

SIR - The future of the South African National Accelerator Centre (NAC) at Faure is indeed under discussion, but we cannot agree with the tenor of Michael Cherry's article (Nature 365, 776; 1993) that the installation is doomed.

The most recent developments are much more positive. Keith Gottschalk and Roger Jardine, both members of the science and technolgy committee of the African National Council (ANC), are to visit NAC to form their own opinions. Although the outcome cannot be predicted, experts from Europe and the United States agree that NAC is outstanding in operational reliability and technological advancement. It is also one of the few particle installations operating a fully integrated hospital dedicated to cancer therapy.

NAC is one of the few facilities in the world at which the benefits of neutrons can be fully exploited, and more than 500 patients have so far been treated. More recently, South African clinicians and engineers have commissioned a protontherapy facility and a unique beampositioning system which is orders of magnitude faster in set-up time than any other system. The radiobiological support from the academic hospitals at Groote Schuur and Tygerberg has led to new insight about beam quality at depth and the necessary filtration. The physical dosimetry group at NAC has made major contributions to beam simulation and the interrelationship between physical and biological effects of high linear energy transfer (LET) neutrons. Increasing use by experts from Belgium, Switzerland, Germany, Japan and the United States demonstrates that NAC already holds a respected position among particle therapy centres.

It is simplistic to suggest that closure of NAC would liberate R34 million for other scientific priorities. The real savings would be much smaller. Salaries at present approximate to R20 million and could not be abruptly stopped. A saving of R10 million would accrue from electricity consumption and general running costs, but this hardly justifies the closure of a successful institution.

NAC provides considerable benefits to South Africa far beyond that of academic training and research. Most of the cancer patients treated at NAC are underprivileged, and could be treated overseas only at enormous cost. Treatment at NAC is free. If NAC were to sacrifice isotope production and research altogether it could treat $200-250$ patients a year. The present figure of 100 patients a year is in line with other dedicated centres with no commitment to other users.

Neutron therapy is an essential part of the radiotherapist's armamentarium, and 5-15 per cent of all patients needing radiotherapy will need neutron therapy. It has been predicted that the United States alone will need 25 proton-therapy installations.

It would be shortsighted if not outright foolish to abandon the first particletherapy facility in the Southern Hemisphere.

Ben Smith

\section{Lothar Böhm}

Radiotherapy Department, Faculty of Medicine,

University of Stellenbosch,

Tygerberg 7505 ,

South Africa

\section{Oiling the waves}

SIR - Daedalus has not carried the idea of using polymeric fullerenes as a lubricant for ships to its logical conclusion (Nature $367,120 ; 1994)$. Why not synthesize a polymeric fullerene that will rotate in only one direction? This would convert the random motion of the water molecules to the ship in a unidirectional fashion, similar to the schemes for exploiting wave motion to generate electricity. Would this be a legal stratagem in boat racing?

Peter S. Tobias

Department of Immunology,

Scripps Research Institute,

10666 North Torrey Pines Road,

La Jolla, California 92037, USA 